\title{
Geolocation, ethics, and HIV research
}

\author{
Renee Garett ${ }^{1}\left(\mathbb{D} \cdot\right.$ Sean D. Young ${ }^{2,3}$
}

Received: 17 June 2021 / Accepted: 8 October 2021 / Published online: 25 October 2021

(c) IUPESM and Springer-Verlag GmbH Germany, part of Springer Nature 2021

\begin{abstract}
The HIV epidemic continues to disproportionally affect marginalized populations. Digital tools, including global positioning system and ecologic momentary assessment, have been studied as methods for improving data collection and interventions among HIV-affected communities. Although people living with HIV and populations at high risk have found it acceptable to use digital technologies for HIV research, concerns over privacy and trust have also been expressed. This paper explores and describes the use of geolocation technology data (e.g., location-based social media) in HIV research as well as the ethical and implementation considerations that warrant examination prior to use. Transparent and clear language in consent forms might improve participant trust in the project and investigators' ability to keep participant data secure and private. With respect to institutional review boards, a committee member who is knowledgeable about digital technologies and consumer protections may offer guidance in assessing adequate protections in study protocols. As technology used in research continues to evolve, investigators and the research community must continue to examine the ethical challenges that emerge to address participant concerns.
\end{abstract}

Keywords Mobility $\cdot$ Ethics $\cdot$ HIV $\cdot$ Informed consent $\cdot$ GPS $\cdot$ Ecologic momentary assessment

\section{Technology in HIV research}

In 2018 , more than 37,000 people were newly diagnosed with HIV in the US, with men who have sex with men (MSM) accounting for $69 \%$ of those diagnoses [1]. Investigators have used a variety of digital tools to study the and address the complexity of this epidemic including social media [2], mobile health [3], ecologic momentary assessment (EMA) [4] and global positioning system (GPS). This paper examines the acceptance of geographic/geolocation data in HIV research and the ethical and implementation considerations surrounding its use for study participants and researchers. (See Fig. 1 as an example of a mapping application created by our team that incorporates HIV-related social media data)

Sean D. Young

syoung5@hs.uci.edu

1 ElevateU, Irvine, CA, USA

2 Department of Emergency Medicine, University of California, Irvine, CA, USA

3 Department of Informatics, University of California Institute for Prediction Technology, University of California, Irvine, CA, USA
A number of studies have recently shown the benefit of location data in improving surveillance for various health issues, including substance use, mental health, COVID19, and HIV [5-10]. In this manuscript, we define location data from technologies to be data from any digital/online technology that collects an individual's location, including social media apps (e.g., Twitter, Facebook), mobile phones (e.g., Apple and Android phones), and other devices that track location. Although limited research has studied how location data might be useful for HIV, there are a number of reasons why location data might help inform HIV surveillance and intervention efforts among people affected by HIV, including helping to provide more contextual and granular information about HIV, improve understanding of structural factors affecting HIV services and their locations, and provide insights into other barriers to HIV prevention and care [11-13].

A number of HIV studies have either incorporated location data into some aspect of the analysis (e.g., using geotagged Twitter data for HIV surveillance) or have explored people's willingness to provide location data. For example, the prevalence of geolocation social networking app use among atrisk populations, including gay, lesbian, or bisexual adults, is higher than their heterosexual counterparts, suggesting 


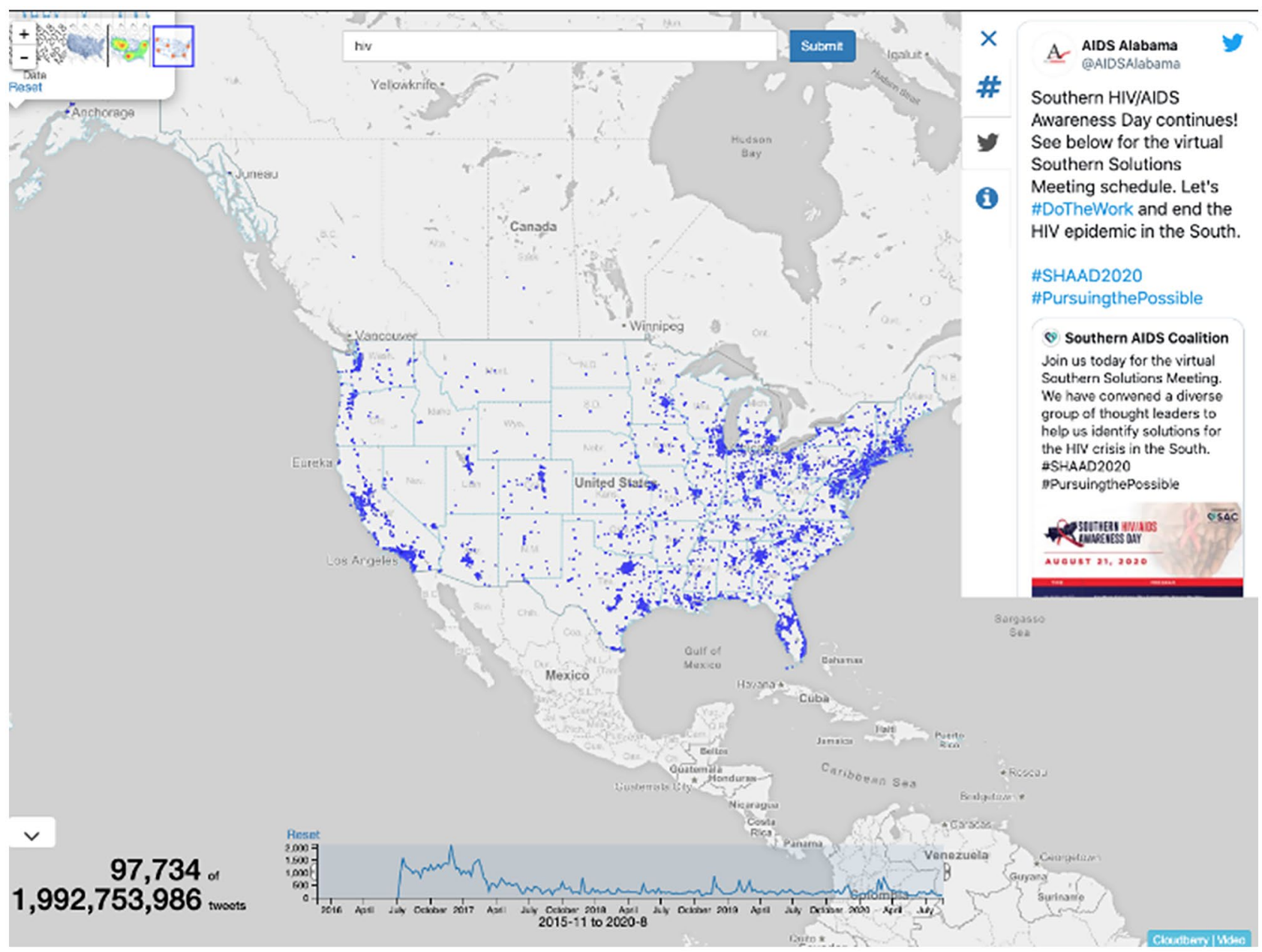

Fig. 1 Sample data from our HIV Map application, which maps the locations of tweets related to HIV content

feasibility and acceptability of using geolocation data for studies among HIV at-risk populations [14]. In fact, studies have already found high feasibility and acceptability of collecting location data among MSM at risk for HIV. In two studies assessing the feasibility and acceptability of a GPS device to track the location of MSM participants, participants in New York [15] and those in Mississippi and Louisiana [16] expressed high comfort in participating in research using GPS that tracked their location.

Feasibility and acceptance of GPS/location data tracking for research have also been explored among people living with HIV. Focus groups and interview data from pregnant and postpartum women living with HIV responded favorably to a conceptual app that used GPS to increase engagement with HIV services [17]. Geologic ecological momentary assessment (GEMA) studies, which combine mobility data with ecological momentary assessment (EMA) data, have also been highly accepted in populations at risk for HIV infection. One study examined geolocation data to provide context to EMA responses to understand health risk environments among youth living in a housing program and homeless youth [18]. Another study assessed the feasibility and acceptability of GEMA among sexual minority men and found high acceptability in this population.

\section{Ethical considerations and challenges}

However, despite acceptability, participants affected by HIV have voiced concerns over privacy in use of location data [19]. Due to the novelty of this research area, most of the work on privacy concerns about location data has been studied in other areas of health [20]. For example, a dementia study found that family and caregivers' most pressing concern about using GPS to track dementia patients was balancing the safety of the patients with their autonomy and privacy [21]. Investigators studying the acceptance of GPS-based research among underrepresented communities in health research found that participant concerns included: unfamiliarity with GPS technology and data gathering methods, 
device safety, privacy and data access, and being tracked [22]. It would be expected that similar findings would occur among HIV-affected populations, especially because of the stigmatization and sensitivity associated with HIV. Future research is needed to explore this area and to identify potential solutions, including policies to protect participants.

Broadly, guidelines such as The Nuremberg Code and Declaration of Helsinki established ethical principles that protect individuals who participate in research studies to ensure autonomy, privacy and confidentiality, and that voluntary consent to participate as a human subject was essential [23]. Although guiding principles exist, institutional ethics committees face challenges in protecting participants, as well as individuals in close proximity to participants engaged in location-based research who did not consent to the study [24]. The constant advancement of technology and novel applications of digital tools leave ethical considerations largely to individual investigators. Additionally, low education populations have low literacy in technicalities of digital tools, understanding of data encryption, and thirdparty transfer of information [25] which may lead to misunderstanding of study protocol or informed consent. Lower health literacy leads to less use of health information technology while higher health literacy leads to decrease trust in technology companies [26] which may affect participation in research studies. Additional ethical challenges regarding research using location tracking include accuracy of data collected and the risk of re-identification of data [25]. To this end, investigators and ethics committees need to examine policies, guidelines, and protocols to ensure the protection of participants, especially those who experience stigma and have historically felt marginalized by the research and medical communities.

\section{Research considerations prior to using location data}

\subsection{Investigators}

Researchers can use technology and apps that are already commercially available or design one to fit their research purpose. If using a commercial app or technology, researchers should read the fine print so they can relay information to participants of where data they provide are going and how companies are using user (participant) data. Studies have shown that users typically do not read End User License Agreements prior to downloading the app on their phone $[27,28]$. Additionally, terms of use agreements are complexly written and often times with language beyond the average readability level [29]. In providing this information to potential participants, researchers provide transparency about the study and foster trust. User mistrust of technology companies may hinder participation if potential participants are not confident in investigators' ability to keep their data private and confidential.

Investigators and research staff should craft the informed consent in language that participants with low literacy can understand. Informed consent written in non-technical language free of jargon may assist participants in truly understanding the purpose of the research and feel confident in giving consent voluntarily. In addition to the types of data being collected, researchers may consider including the types of data it will not collect (i.e., conversations) and the limitations of the research protocol in data collection. Although ubiquity of mobile phones, GPS, and health apps lend general familiarity of these technologies, new digital tools are constantly developed with new features that may seem obscure to potential participants leading to uncertainty about the data collection process.

In publications, researchers provide study details for the purposes of transparency with the scientific community and for possible replication. Previous investigators using GPS technology among MSM provided detail about the technology used, staff training on the technology, and where data were stored [15]. These details allowed for readers to be thoughtfully informed of the research process. In addition to details about the technology used, investigators may want to include informed consent procedure in published manuscripts to inform other investigators of best practices.

\subsection{Institutional review boards}

Institutional Review Boards (IRB) were established to regulate ethical conduct of research with the primary aim of reviewing research protocols and ensuring the safety and welfare of human participants [23]. Composition of IRB includes individuals with experience and expertise in the research field the board reviews to amply provide safeguards for participants [30]. In aligning with this requirement, it would behoove IRBs to include an individual, whether it is someone whose primary concerns are in the scientific or non-scientific areas, with experience and expertise in digital technologies to provide guidance of how such technologies are used in the general population and its application in research. Members with technical knowledge of operations as well as current laws and policies about privacy and consumer protections may be able to guide the committee in assessing if protocols provide sufficient information in the consent form and its language. As technology constantly evolves and more research is conducted using digital tools to recruit participants, collect data, and implement intervention, individuals with an understanding of technologies, beyond that of a typical consumer, may impart perspectives 
about participant safety, privacy and confidentiality concerns, and data storage.

IRBs may also play a role in facilitating a collaboration between researchers and the communities they intend to study. In some cases, investigators provide justification for procedures in the application (i.e., number of potential participants recruited, use of specific instruments, translation of survey questionnaire, recruitment material). IRBs can inquire from investigators if they collaborated with the MSM community in the design of their survey, informed consent, recruitment materials, and to what extent their protocols were informed from this partnership. In dealing with a nascent technology such as EMA in this community, participants recommended that investigators highlight working with MSM in several aspects of the research design, such as crafting the informed consent, to increase buy-in from the community [31].

\section{Conclusion}

Feasibility and acceptance of devices used to track participant location in research is high among populations at risk for HIV infection and those living with HIV. However, because of the potential ongoing ethical concerns with these approaches, transparency about study objectives, protocols, and risk management are crucial to communicate with potential participants. Both investigators and ethics committees have an integral role in ensuring participant autonomy and safety, especially for a population that continues to face stigma. As digital technologies will continue to evolve, investigators will find novel applications for them to mitigate the HIV epidemic, which necessitates an unceasing effort to revisit ethical challenges that affect research conduct.

Authors' contributions All authors contributed to the manuscript conception and design, and read and approved the final manuscript.

Funding This study was funded by grants from the National Institute of Mental Health [NIMH, MH106415], National Institute on Drug Abuse (NIDA), National Institute of Allergy and Infectious Diseases (NIAID), and National Center for Complementary and Integrative Health (NCCIH).

Data availability Not applicable.

Code availability Not applicable.

\section{Declarations}

Ethics approval Not applicable.
Informed consent Not applicable.

Conflicts of interest The authors have no conflicts of interest to declare that are relevant to the content of this article.

\section{References}

1. Centers for Disease Control and Prevention. HIV Surveillance Report, 2018 (Updated). 2020. https://www.cdc.gov/hiv/library/ reports/hiv-surveillance/vol-31/index.html (accessed 10 Jun 2020).

2. Cao B, Gupta S, Wang J, et al. Social media interventions to promote HIV testing, linkage, adherence, and retention: systematic review and meta-analysis. J Med Internet Res. 2017;19:e394. https://doi.org/10.2196/jmir.7997.

3. Daher J, Vijh R, Linthwaite B, et al. Do digital innovations for HIV and sexually transmitted infections work? Results from a systematic review (1996-2017). BMJ Open. 2017;7. https://doi. org/10.1136/bmjopen-2017-017604.

4. Smiley SL, Milburn NG, Nyhan K, et al. A systematic review of recent methodological approaches for using ecological momentary assessment to examine outcomes in U.S. based HIV research. Curr HIV/AIDS Rep. 2020;17:333-42. https://doi.org/10.1007/ s11904-020-00507-0.

5. Badr HS, Du H, Marshall M, et al. Association between mobility patterns and COVID-19 transmission in the USA: a mathematical modelling study. Lancet Infect Dis. 2020;20:1247-54. https://doi. org/10.1016/S1473-3099(20)30553-3.

6. Depp CA, Bashem J, Moore RC, et al. GPS mobility as a digital biomarker of negative symptoms in schizophrenia: a case control study. npj Digital Medicine. 2019;2:1-7. https://doi.org/10.1038/ s41746-019-0182-1.

7. Young SD, Zheng K, Chu LF, et al. Internet searches for opioids predict future emergency department heroin admissions. Drug Alcohol Depend. 2018;190:166-9. https://doi.org/10.1016/j.drugalcdep. 2018.05.009.

8. Zhang Q, Chai Y, Li X, et al. Using internet search data to predict new HIV diagnoses in China: a modelling study. BMJ Open. 2018;8:e018335. https://doi.org/10.1136/bmjopen-2017-018335.

9. Young SD, Rivers C, Lewis B. Methods of using real-time social media technologies for detection and remote monitoring of HIV outcomes. Prev Med. 2014;63:112-5. https://doi.org/10.1016/j. ypmed.2014.01.024.

10. Melvin S, Jamal A, Hill K, et al. Identifying sleep-deprived authors of tweets: prospective study. JMIR Mental Health. 2019;6:e13076. https://doi.org/10.2196/13076.

11. Felker-Kantor E, Polanco C, Perez M, et al. Daily activity spaces and drug use among female sex workers living with HIV in the Dominican Republic. Health Place. 2021;68:102527. https://doi. org/10.1016/j.healthplace.2021.102527.

12. Benbow N, Kirkpatrick C, Gupta A, et al. An iterative process of integrating and developing big data modeling and visualization tools in collaboration with public health officials. Sage Research Methods Cases In Press.

13. Young SD, Torrone EA, Urata J, et al. Using search engine data as a tool to predict syphilis. Epidemiology. 2018;29:574-8. https:// doi.org/10.1097/EDE.0000000000000836.

14. Pew Research Center. 10 facts about Americans and online dating. Pew Research Center. 2020.https://www.pewresearch.org/ fact-tank/2020/02/06/10-facts-about-americans-and-online-dating/ (accessed 26 Mar 2021).

15. Duncan DT, Kapadia F, Regan SD, et al. Feasibility and acceptability of global positioning system (GPS) methods to study the spatial contexts of substance use and sexual risk behaviors among 
young men who have sex with men in New York City: a P18 cohort sub-study. PLoS One. 2016;11. https://doi.org/10.1371/ journal.pone.0147520.

16. Duncan DT, Chaix B, Regan SD, et al. Collecting mobility data with GPS methods to understand the HIV environmental riskscape among young black men who have sex with men: a multi-city feasibility study in the Deep South. AIDS Behav. 2018;22:3057-70. https://doi.org/10.1007/s10461-018-2163-9.

17. Clouse K, Phillips TK, Mogoba P, et al. Attitudes toward a proposed GPS-based location tracking smartphone app for improving engagement in HIV care among pregnant and postpartum women in South Africa: focus group and interview study. JMIR Form Res. 2021;5:e19243. https://doi.org/10.2196/19243.

18. Henwood BF, Redline B, Dzubur E, et al. Investigating health risk environments in housing programs for young adults: protocol for a geographically explicit ecological momentary assessment study. JMIR Res Protoc. 2019;8. https://doi.org/10.2196/12112.

19. Duncan DT, Park SH, Goedel WC, et al. Acceptability of smartphone applications for global positioning system (GPS) and ecological momentary assessment (EMA) research among sexual minority men. PLoS One. 2019;14. https://doi.org/10.1371/journal.pone. 0210240.

20. Romero R, Young SD. Ethical perspectives on data sharing among patients before and immediately after the onset of COVID-19 in the United States. Ethics \& Behavior Published Online First: In Press. https://doi.org/10.1080/10508422.2021.1884079.

21. Landau R, Auslander GK, Werner S, et al. Families' and professional caregivers' views of using advanced technology to track people with dementia. Qual Health Res. 2010;20:409-19. https:// doi.org/10.1177/1049732309359171.

22. Nebeker C, Murray K, Holub C, et al. Acceptance of mobile health in communities underrepresented in biomedical research: barriers and ethical considerations for scientists. JMIR Mhealth Uhealth. 2017;5:e87. https://doi.org/10.2196/mhealth.6494.

23. Corrigan O. Empty ethics: the problem with informed consent. Sociol Health Illn. 2003;25:768-92. https://doi.org/10.1046/j. 1467-9566.2003.00369.x.
24. Nebeker C, Linares-Orozco R, Crist K. A multi-case study of research using mobile imaging, sensing and tracking technologies to objectively measure behavior: ethical issues and insights to guide responsible research practice. J Res Adm. 2015;46:118-37.

25. Apte $\mathrm{A}$, Ingole $\mathrm{V}$, Lele $\mathrm{P}$, et al. Ethical considerations in the use of GPS-based movement tracking in health research - lessons from a care-seeking study in rural west India. J Glob Health. 2019;9. https://doi.org/10.7189/jogh.09.010323.

26. Mackert M, Mabry-Flynn A, Champlin S, et al. Health literacy and health information technology adoption: the potential for a new digital divide. J Med Internet Res. 2016;18:e264. https://doi.org/ 10.2196/jmir.6349.

27. Deloitte. 2017 Global Mobile Consumer Survey: US edition. 2017.

28. Shklovski I, Mainwaring SD, Skúladóttir HH, et al. Leakiness and creepiness in app space: perceptions of privacy and mobile app use. In: Proceedings of the 32nd annual ACM conference on Human factors in computing systems - CHI '14. Toronto, Ontario, Canada: ACM Press 2014;2347-56. https://doi.org/10.1145/2556288.2557421.

29. Benoliel U, Becher SI. The duty to read the unreadable. Rochester, NY: Social Science Research Network 2019. https://doi.org/10. 2139/ssrn.3313837.

30. Moon MR. The history and role of institutional review boards: A useful tension. AMA J Ethics. 2009;11:311-6. https://doi.org/10. 1001/virtualmentor.2009.11.4.pfor1-0904.

31. Hubach RD, O'Neil A, Stowe M, et al. Perceived confidentiality risks of mobile technology-based ecologic momentary assessment to assess high-risk behaviors among rural men who have sex with men. Arch Sex Behav Published Online First: 20 February 2020. https://doi.org/10.1007/s10508-019-01612-x.

Publisher's Note Springer Nature remains neutral with regard to jurisdictional claims in published maps and institutional affiliations. 